\title{
Diffusive Capture Process on Complex Networks
}

\author{
Sungmin Lee, Soon-Hyung Yook $⿴$ and Yup Kin円 \\ Department of Physics and Research Institute for Basic Sciences, Kyung Hee University, Seoul 130-701, Korea
}

(Dated: September 3, 2018)

\begin{abstract}
We study the dynamical properties of a diffusing lamb captured by a diffusing lion on the complex networks with various sizes of $N$. We find that the life time $\langle T\rangle$ of a lamb scales as $\langle T\rangle \sim N$ and the survival probability $S(N \rightarrow \infty, t)$ becomes finite on scale-free networks with degree exponent $\gamma>3$. However, $S(N, t)$ for $\gamma<3$ has a long-living tail on tree-structured scale-free networks and decays exponentially on looped scale-free networks. It suggests that the second moment of degree distribution $\left\langle k^{2}\right\rangle$ is the relevant factor for the dynamical properties in diffusive capture process. We numerically find that the normalized number of capture events at a node with degree $k, n(k)$, decreases as $n(k) \sim k^{-\sigma}$. When $\gamma<3, n(k)$ still increases anomalously for $k \approx k_{\max }$. We analytically show that $n(k)$ satisfies the relation $n(k) \sim k^{2} P(k)$ and the total number of capture events $N_{\text {tot }}$ is proportional to $\left\langle k^{2}\right\rangle$, which causes the $\gamma$ dependent behavior of $S(N, t)$ and $\langle T\rangle$.
\end{abstract}

PACS numbers: 05.40.Fb,89.75.Hc,89.75.Fb

\section{INTRODUCTION}

The diffusion-controlled reactions, in which diffusing particles are immediately converted to a product if a pair of them meets together, have many physical applications. Examples include electron trapping and recombination, exciton fusion, wetting, melting, and commensurateincommensurate transitions [1, 2, 3, 4, 5, 6, 7]. Among these examples, dynamical properties of wetting, melting, and commensurate-incommensurate transition are known to be related to the diffusive capture process [3, 4, 5, 6, 7], whose kinetics can be simplified by diffusing preys-predators model $[\underline{8}, 9]$.

In general, the dynamical properties of diffusioncontrolled reactions are closely related to the first-passage phenomena of random walks (RWs), because the reaction occurs when diffusing particles first meet a trap or an absorbing boundary [7, 8, 9]. Especially, the diffusive capture process can be mapped into coupled RWs 10, in which $n$ predator random walkers (lions or moving trap) stalk a prey random walkers (a lamb or a moving particle). Since the first-passage probability can be easily obtained from the survival probability [7], the survival probability of a diffusing particle or a lamb is important to understand long time characteristics of the diffusive capture process [8, 9]. The survival probability of a lamb has been well investigated in $d$-dimensional hypercubic lattice. The diffusive capture process in the spatial dimension $d>2$ is unsuccessful and the survival probability is finite for any $n$. In $d=2$, the capture is successful but not efficient, i.e., the life time of a prey random walker is infinite and the survival probability shows a logarithmic decay [11]. The predators efficiently capture a prey for spatial dimension $d<2$. On a $d$-dimensional regular lattice, the survival probability $S(t)$ depends on

*Electronic address: syook@khu.ac.kr

†Electronic address: ykim@khu.ac.kr $d$ as follows $[8,9,10,12,13$.

$$
S(t) \sim \begin{cases}t^{-\beta} & \text { for } d<2 \\ (\ln t)^{-n} & \text { for } d=2 \\ \text { finite } & \text { for } d>2,\end{cases}
$$

where the exponent $\beta$ varies as $n$ and the ratio of diffusion constant of walks.

There have been increasing interests on complex networks, since many structures of physically interacting systems are shown to form nontrivial complex networks 14, 15, 16, 17, 18, 19]. The early studies on complex networks mainly focused on their topological properties. Recently, the physical systems whose elements interact along the links in complex networks and the effects of the underlying topologies on the dynamical properties of the system have drawn much attention. Many dynamical systems on complex networks show rich behaviors which are far from the mean-field expectations 20, 21, 22, 23]. Recently, RWs on complex networks have been studied to show that the dynamical properties of RWs are closely related to the topology of underlying networks 24, 25]. This implies that the first-passage property of the diffusive capture process is also affected by the topology of underlying network. Moreover, the several studies on RWs have provided very interesting application to searching information over the Internet [26, 27]. For example, the major searching engines such as Google and YAHOO use general random walking robots along the links between hyper-texts to collect information of each web page [28]. Some peer-to-peer $(\mathrm{P} 2 \mathrm{P})$ network protocols also exploit the dynamical properties of RWs on complex networks [26]. Especially, the searching algorithm for P2P network can be mapped to the system of a diffusing particle to find an immobile absorbing particle (static trap). Our recent study [27] has also found that the searching algorithm inspired by the diffusive capture process can dramatically reduce the traffic over the Internet with scalable searching time. The diffusive capture process can be also applied to investigating condensed phase of conserved-mass aggregation model on scale-free networks (SFNs) with 
$2<\gamma \leq 3[29]$. Therefore, it is theoretically and technologically very interesting to study the effect of nontrivial complex networks on the diffusive capture process.

In order to understand the relationship between the non-trivial topology of underlying network and the diffusive capture process on it, we study the random walking lamb and lions on SFNs. The SFNs are the networks whose degree distribution follows $P(k) \sim k^{-\gamma}$. The degree exponent $\gamma$ of the most natural and technological networks is in the range $2<\gamma \leq 3$ [15, 30]. Moreover, the probability that a random walker visits a node has been shown to be proportional to the degree $k$ of the node 24]. So, the probability that two random walking particles meet at a node is proportional to $k^{2}$. Therefore, we expect that the dynamical properties of the diffusive capture process on the SFNs with the degree exponent $2<\gamma \leq 3$ are different from those on the SFNs with $\gamma>3$, because $\left\langle k^{2}\right\rangle$ diverges for $\gamma<3$. To verify our expectations, we study the diffusive capture process on the SFNs. More recently, Noh et al. 25] also showed that the return probability of a random walker on the looped SFNs (LSFNs) is completely different from that on the tree-structured scale-free networks (TSFNs). Therefore, the diffusive capture process on TSFNs is also investigated.

This paper is organized as follows: In Sec. II, we present a method of generating TSFNs and dynamical properties of the diffusive capture process on TSFNs. The results on LSFNs are shown in Sec. III. In Sec. IV, we discuss the relation between degrees of SFNs and capture events. Finally we summarize our results in Sec. V.

\section{DIFFUSIVE CAPTURE PROCESS ON TSFNS}

All the models are defined on a graph $G r=\{N, K\}$, where $N$ is the number of nodes in the networks and $K$ is the number of degrees with the average degree $\langle k\rangle=$ $2 K / N$. Networks with the sizes $N=10^{3} \sim 10^{6}$ are generated for each $\gamma$.

To make tree structure, the total number of links in networks with the size $N$ is constrained to be $K_{T}=2(N-1)$. So we use the following method to make TSFNs. Select $N$ integer random numbers $\left\{k_{1}, k_{2}, \cdots, k_{N}\right\}$ whose probability distribution satisfies $f(k) \sim k^{-\gamma}$. Let $K_{0}=\sum_{i=1}^{N} k_{i}$. If $K_{T}>K_{0}\left(K_{T}<K_{0}\right)$, then choose $k_{j}$ randomly from the set $\left\{k_{i}\right\}$ and decrease (increase) by 1 as $k_{j} \rightarrow k_{j}+1\left(k_{j} \rightarrow k_{j}-1\right)$. If the resulting $k_{j}$ becomes 0 , the above process is rejected and take the same process again. This process is repeated until $K_{T}=K_{0}$ is fulfilled. Sort the final set $\left\{k_{1}, k_{2}, \cdots, k_{N}\right\}$ with $K_{T}=\sum_{i=1}^{N} k_{i}$ to satisfy $k_{1}>k_{2}>\cdots>k_{N}$ and set $k_{i}$ to be the degree of the node $i$. Now link the nodes $2,3, \cdots, k_{1}+1$ to the node 1 to make the degree of the node 1 be $k_{1}$. Next, link the nodes $k_{1}+2, k_{1}+3, \cdots$, $k_{1}+k_{2}$ to the node 2 to make the degree of the node 2 be $k_{2}$. Repeat the same processes until no isolated node remains.

Figs. 1(a) and 1(b) show snapshots of typical networks generated by the above procedure with 1000 nodes. Fig. 1(c) shows the degree distribution of TSFNs constructed with $\gamma=2.4$ and 4.3. To generate TSFNs we use $N=10^{5}$. The data show a good agreement between the exponents $\gamma$ obtained from the degree distribution, $P(k) \sim k^{-\gamma}$, of the constructed network (symbols) and $\gamma$ of the original distribution $f(k) \sim k^{-\gamma}$ (solid and dashed lines). As shown in Figs. 1(a) and 1 (b), the topological structure of TSFNs with $\gamma>3$ is completely different from that with $\gamma<3$. We find relatively small degree fluctuation in the networks with $\gamma>3$ (see Fig. 1) (a)). The small fluctuation causes almost homogeneous topological properties. Thus, we expect that the dynamical properties on the networks with $\gamma>3$ follow the meanfield expectations, i.e., the non-zero survival probabilities of a lamb in $N \rightarrow \infty$ limit. However, for network with $\gamma<3$, there exist many short branches and a big hub having most links. The dynamical properties of a given system on this kind of network can be dominated by this hub. Therefore, as we shall show later, the lamb can easily hide from the lions by moving to a different branch through the hub.

Now, let's discuss the dynamical properties of the diffusive capture process on the constructed TSFNs. Initially the lamb and $n$ lions are randomly distributed to the nodes on the networks. In the simulations, we consider two updating rules to determine the position of the random walking lamb and lions: 1) parallel update and 2) random update. In the parallel update rule, we move all lions and a lamb at the same time, then check if the lamb meets the lion(s). In this algorithm, the lamb and the lions should be initially separated by $2 m(m=1,2, \cdots)$ steps. Otherwise, the lamb never meets the lion moving on TSFNs. In the random update rule, we choose one among the lamb or lions at random. The randomly chosen lamb or lion takes a random walk and then check if the lamb meets the lion. The unit time is defined by $(n+1)$ such random movements. We find that there is no significant difference between dynamical behaviors for each update rule. We also find that the dynamical behaviors are not varied critically if $n$ is varied. Therefore, we will mainly show the simulation results for 1 lamb and 10 lions in this article. All the numerical results are obtained by averaging over 100 network realizations and 1000 histories for each network realization.

Fig. 2 shows the plot of the average life time $\langle T\rangle$ on TSFNs with various $\gamma$ against $N$ using the random update rule. $\langle T\rangle$ increases with $N$ for any $\gamma$. The lines represent the best fits of the relation $\langle T\rangle \sim N^{\alpha}$ to the data. From the fits, we find that $\langle T\rangle$ follows the powerlaw $\langle T\rangle \sim N^{\alpha}$ with $\alpha=1$ for $\gamma \geq 3$, and $\alpha=0.7$ for $\gamma=2.4$ (dotted line). But, as shown in the Fig. $2\langle T\rangle$ for $\gamma=2.4$ deviates substantially from the power-law relation $\langle T\rangle \sim N^{0.7}$ as increasing $N$ (square). The inset shows the results obtained by using the parallel update 
rule. The data obtained from the parallel update rule show nearly the same dynamical behaviors as those obtained from the random update rule.

We also measure the survival probability on TSFNs with various $\gamma$ and $N$. Fig. [3] shows $S(N, t)$ for $\gamma=4.3$ (a), 3.0 (b) and 2.4 (c) from the random update rule. As is shown in Fig. 3] the survival probability for $\gamma \geq 3$ exponentially decays as $S(N, t) \sim \exp (-t / \tau(N))$, however, $S(N, t)$ for $\gamma<3$ manifests a substantial deviation from an exponential decay. If we assume that $S(N, t)=S_{o} e^{-t / \tau}$, then we obtain $\langle T\rangle \sim \tau(N)$ from the relation $\langle T\rangle=\int_{0}^{\infty} t(-d S(N, t) / d t) d t$. Therefore, the characteristic time $\tau$ of $S(N, t)$ scales in the same manner as $\langle T\rangle$, and the scaling $t / N$ collapses $S(N, t)$ 's for various $N$ into one curve when $\gamma \geq 3$ [see Fig. [3(a), (b)]. The relation $\tau(N) \sim N$ implies that $S(N, t)$ becomes a nonzero constant in the limit $N \rightarrow \infty$. Therefore, the exponentially decaying $S(N, t)$ for $\gamma>3$ can be regarded as the finite size effect. However, $S(N, t)$ for TSFN with $\gamma=2.4$ shows the long-living tail [Fig. 33 (c)] which decreases more slowly than exponential decay. Hence, we expect

$$
\lim _{t \rightarrow \infty} \lim _{N \rightarrow \infty} S(N, t)= \begin{cases}\text { long-living tail } & (\gamma<3) \\ \text { finite } & (\gamma \geq 3) .\end{cases}
$$

The origin of $\gamma$ dependent behaviors of $S(N, t)$ will be discussed in Sec. IV.

We now discuss the origin of the long-living tail of $S(N, t)$ for $\gamma=2.4$. One possible explanation of the slowly decaying tail of $S(N, t)$ can be understood from the topological properties of TSFNs. As shown in Fig. [1 (b), the biggest hub in the TSFN with $\gamma<3$ has many short branches. Hence, if the lamb hides from the lions by moving into a different branch, then the lion should visit almost all branches to search for a hidden lamb. Thus, the hidden lamb can live longer than the one in the same branch with the lion. Therefore, $S(N, t)$ on TSFN with $\gamma<3$ can have the long-living tail. In order to verify this argument, we count the number of capture events $N(L, T)$ with the life time $T$ among $10^{6}$ different histories on TSFN with $\gamma=2.4$ (Fig. [4) by using the random update rule. At the same time we also measure the maximum separation $L$ between the lamb and the lion during random walks. Initially we set the shortest path length $l$ between the lamb and lion to be 2 . We use the TSFN whose average diameter $\bar{d}$ is $\bar{d} \simeq 4$. If $L \simeq \bar{d}$, then the lamb and the lion have once been in different branches during random walks. The data in Fig. 4 explicitly shows that $N(L, t)$ for $L=2$ decays fast (the maximum life time of a lamb is $\left.T_{\max } \simeq 10^{2}\right)$. In contrast, $N(L, t)$ for $L=4$ shows the long-living tail up to $T_{\max } \simeq 10^{4}$. From the relation $S(t) \propto-\int_{t} \sum_{L} N(L, t) d t$, one can easily see that the long-living tail of $S(t)$ is caused by the diffusive capture process with $L=4$.

\section{DIFFUSIVE CAPTURE PROCESS ON LSFNS}

The dynamical behavior of one lamb and one lion model with the random update rule on LSFNs has already been reported in Ref. 29]. We measure the $S(N, t)$ and $\langle T\rangle$ for various $n$ 's with the random and the parallel update algorithms. We check that $\langle T\rangle$ and $S(N, t)$ scale in the same manner with those reported in Ref. [29]. Therefore, we will report our results briefly in this section. To generate the LSFNs of sizes $N=10^{3} \sim 10^{6}$, we use the static network model suggested by Ref. 31 with $\langle k\rangle=4$. In Fig. 5 we display $S(N, t)$ for $n=10$ on LSFNs with various $\gamma(=4.3,3.0$, and 2.4). $S(N, t)$ exponentially decays as $S(N, t) \sim \exp (-t / \tau)$ for all $\gamma$ 's. As discussed in Sec. II, by assuming that $S(N, t) \sim$ $\exp (-t / \tau)$, we obtain the relation $\langle T\rangle \sim \tau(N)$. Using the value of $\alpha$ 's obtained from the best fit of $\langle T\rangle \sim N^{\alpha}$ to the data (which are not shown), we scale the time as $t / N$ for $\gamma>3$ and as $t / N^{0.55}$ for $\gamma=2.4$. As shown in Fig. 囵(a), $S(N, t)$ for $\gamma>3$ collapses well into a single curve. This results implies that $\tau(N) \sim N$ for $\gamma>3$. However, as we can see in Fig. [5 (c), $S(N, t)$ for $\gamma=2.4$ does not collapse into a single curve by the simple scaling. Note that $\gamma=3$ is marginal below which $\left\langle k^{2}\right\rangle$ diverges. Thus, the logarithmic correction for the scaling of $\tau$ can be expected for $\gamma=3$. In Fig. [5 (b), we scale the time as $t / N(\log N)^{-1.6}$ and find that $S(N, t)$ for $\gamma=3$ collapses into one curve. This means that $\tau$ scales as $\tau \sim N(\log N)^{-1.6}$. Therefore, we expect

$$
\lim _{t \rightarrow \infty} \lim _{N \rightarrow \infty} S(N, t)= \begin{cases}S_{o} e^{-t / \tau_{\infty}} & (\gamma<3) \\ \text { finite } & (\gamma \geq 3),\end{cases}
$$

where $\tau_{\infty}$ is the asymptotic value of $\tau$, and depends on $\gamma$. Eqs. (2) and (3) show one of the evidences that the dynamical behavior of the diffusive capture process is related to $\left\langle k^{2}\right\rangle$

\section{RELATION BETWEEN DEGREES AND CAPTURE EVENTS}

In the previous sections, we show that the underlying topology significantly affects the dynamical behaviors of $S(N, t)$ and $\langle T\rangle$ of a lamb. For the quantitative description of the relationship between the structure of the networks and the dynamical properties of lamb-lion model, we count the capture events occurring at the nodes with degree $k$. Fig. 6(a) shows the normalized number of capture events $n(k)$ on LSFNs with 1-lamb and 10-lions. As we can see, $n(k)$ 's both on LSFNs and TSFNs with $\gamma=4.3$ decay as $n(k) \sim k^{-\sigma}$ and the obtained $\sigma$ is $\sigma \simeq 2.3$. However, $n(k)$ measured on LSFNs with $\gamma=2.4$ decreases with $\sigma \simeq 0.4$ for $k<10^{3}$ and increases with $\sigma \simeq-1.35$ for $k>10^{3}$. On TSFNs with $\gamma=2.4$, we also find $\sigma \simeq 0.4$ for $10^{2}<k<10^{3}$ and $\sigma \simeq-1.9$ for $k>10^{3}$ (see Fig. 6(b)). The decreasing behavior of $n(k)$ 
for moderate $k$ can be explained by the following analysis. In the steady-state, the probability that a random walker visits a node $i$ with degree $k_{i}$ is given by [24]

$$
P_{i}^{\infty}=A k_{i}
$$

where $A=1 / \sum_{i} k_{i}$. Therefore, the normalized capture events simply can be written as

$$
n(k)=\sum_{i, j=1}^{N} P_{i}^{\infty} \delta_{k_{i}, k} P_{j}^{\infty} \delta_{i, j}=A^{2} k^{2} N P(k) .
$$

If $P(k) \sim k^{-\gamma}$, then we obtain

$$
\sigma=\gamma-2
$$

Since we do not assume any topological property of underlying networks, Eq. (6) is valid for any SFNs and the obtained values of $\sigma$ from the simulations for $k<10^{3}$ is consistent with Eq. (6). Note that the $n(k)$ 's anomalously increase for $k>10^{3}$ both on the LSFNs and the TSFNs with $\gamma=2.4$. This anomalous behavior of $n(k)$ can be explained by the rarity of the dominant hubs in complex networks, i.e., the $P(k)$ for $k \approx k_{\max }$ does not change much as $k$ varies. Here $k_{\max }$ is the degree of the biggest hub. Thus, if we assume that $P\left(k \approx k_{\max }\right) \sim$ const., then $n(k)$ increases as $n(k) \sim k^{2}$ or

$$
\sigma=-2
$$

for $k \approx k_{\max }$. The obtained $\sigma\left(k \approx k_{\max }\right)$ 's for $\gamma=2.4$ from the numerical simulations are -1.35 on LSFN and -1.9 on TSFN. The value of $\sigma\left(k \approx k_{\max }\right)$ obtained from the simulation on LSFN with $\gamma=2.4$ is considerably smaller than Eq. (7). This disagreement might be originated from our assumption that $P\left(k \approx k_{\max }\right)$ does not depend on $k$. For example, if we assume that $P\left(k \approx k_{\max }\right) \sim k^{-\epsilon}$ with $\epsilon<2$, then $\sigma$ becomes $\sigma=\epsilon-2(<0)$. Eqs. (5) and (7), thus, provide the topological origin of the gathering behavior of random walkers at the dominant hubs. It implies that the lamb and the lion have a strong tendency to move into big hubs, i.e. the hubs collects the lamb and the lion. Therefore, the hubs in the networks with strong heterogeneity become the effective attractors of random walking lamb and lions.

Another important consequence of Eq. (5) is that the dynamical properties of lamb-lion model depend on $\left\langle k^{2}\right\rangle$. Note that from Eq. (5) the total number of capture event $N_{\text {tot }}$ can be expressed as

$$
N_{t o t} \propto \sum_{k} n(k) \sim \sum_{k} k^{2} P(k) \sim\left\langle k^{2}\right\rangle .
$$

If $\gamma<3$ then $N_{\text {tot }}$ also diverges. This means that the lions on the networks can capture the lamb no matter how the lamb is trying to escape from the lions. Therefore, $S(N, t) \rightarrow 0$ in the limit $N \rightarrow \infty$. For $\gamma>3, N_{t o t}$ converges to a finite value and the lions can catch the lamb with a probability $p<1$, thus $S(N, t) \neq 0$ for large $N$. The expectations agree well with our simulation results shown in Sec. II and III. Based on Eq. (8), we also expect that the long-living tail of $S(N, t)$ on TSFN with $\gamma<3$ eventually goes to 0 .

\section{SUMMARY AND DISCUSSION}

We investigate the diffusive capture process on two different kinds of SFNs. The diffusive capture process can be mapped into the diffusing lamb and lion model. We measure $S(N, t)$ and $\langle T\rangle$ of a lamb. We find that on both TSFNs and LSFNs $S(N, t)$ of a lamb decays exponentially for $\gamma>3$. Simple scaling arguments predict that the exponentially decaying $S(N, t)$ for $\gamma>3$ is originated from the finite size effect. This result recovers the meanfield expectation $S(N, t) \rightarrow$ finite in the limit $N \rightarrow \infty$. However, we find that $S(N, t)$ on TSFNs with $\gamma<3$ has the long-living tail. By measuring the number of capture events with life time $T$ we find that the long-living tail is caused by enormously many short branches in TSFNs. On LSFN with $\gamma<3$, we find that $S(N, t)$ decays exponentially and no lamb can survive in thermodynamic limit.

In order to understand the non-mean-field behavior of $S(N, t)$ for $\gamma<3$, we study the normalized number of capture events $n(k)$ both numerically and analytically. From the numerical simulations, we find that $n(k)$ decreases as a power-law, $n(k) \sim k^{-\sigma}$. The simple analytic derivation of $n(k)$ provides the relation $\sigma=\gamma-2$, which shows a good agreement with numerical results for moderate $k$. However, we find that $n(k)$ anomalously increases for large $k$ in the simulations. This means that the most of the lambs are captured by lions at the hubs. Hence, those hubs become the effective attractors. By assuming the rarity of dominant hubs and $P(k) \approx$ const. for large $k\left(\approx k_{\max }\right)$, we show that the $n(k)$ can increase for large $k$. From the analytic expectation of $n(k)$, we also show that $N_{t o t} \sim\left\langle k^{2}\right\rangle$. This implies that if the fluctuation in degree is large enough $(\gamma<3)$ then the dynamical behaviors of the diffusive capture process deviate from the mean-field expectations by emerging effective attractors of random walkers. From the analytic expectation of $n(k)$ and $N_{t o t}$, we also expect that the long-living tail of $S(N, t)$ observed on TSFN with $\gamma<3$ (see Eq. (2) ) will eventually go to 0 due to the diverging $\left\langle k^{2}\right\rangle$.

This work is supported by Korea Research Foundation Grant No. KRF-2004-015-C00185. 
[1] M. Agranovich and M. D. Galanin "Electronic Energy Transfer in Condensed Matter" (North-Holland, Amsterdam, 1985).

[2] S. J. Parus and R. Kopelman, Phys. Rev. B 39, 889 (1989).

[3] D. ben-Avraham, J. Chem. Phys. 88941 (1988).

[4] M. E. Fisher, J. Stat. Phys. 34, 667 (1984).

[5] D. E. Huse and M. E. Fisher Phys. Rev. B 29, 239 (1984).

[6] R. Lipowsky and T. M. Nieuwenhuizen, J. Phys. A: math. gen. 21, L89 (1988).

[7] S. Redner, "A Guide to First-Passage Processes" (Cambridge University Press, New York, 2001).

[8] P. L. Krapivsky and S. Redner, J. Phys. A 29, 5347 (1996).

[9] S. Redner and P. L. Krapivsky, Am. J. Phys. 67, 1277 (1999).

[10] M. Bramson and D. Griffeath, "Capture problems for coupled random walks", in Random walks, Brownian motion, and interacting particle systems: a festschrift in honor of Frank Spitzer, eds. R. Durrett and H. Kesten, (Boston, Birkhauser, 1991), 153-188.

[11] G. H. Weiss, Aspects and Applications of the Random walk (North-Holland, Amsterdam, 1994).

[12] W. Feller, An Introdution to Probability Theory (Wiley, New Yourk, 1971), Vol. 1.

[13] Aspects and Applications of the Random Walk (NorthHolland, Amsterdam, 1994).

[14] H. Jeong, B. Tombor, R. Albert, Z.N. Oltvai, and A.L. Barabási, Nature 407, 651 (2001); S.-H. Yook Z.N. Oltvai, and A.-L. Barabási, Porteomics 4, 928 (2004).

[15] S. N. Dorogovtsev and J. F. F. Mendes, Adv. Phys. 51, 1079 (2002); R. Albert and A.-L. Barabási, Rev. Mod. Phys. 74, 47 (2002).

[16] R. Albert, H. Jeong and A.-L. Barabási, Nature (London) 401, 130 (1999).

[17] A. Broder, R. Kumar, F. Maghoul, P. Raghavan, S. Rajalopagan, R. Stata, A. Tomkins and J. Wiener, Comput. Netw. 33, 309 (2000).

[18] M. Faloutsos, P. Faloutsos and C. Faloutsos, Comput. Commun. Rev. 29, 251 (1999).

[19] S.-H. Yook, H. Jeong and A. -L. Barabási, PNAS 99, 13382 (2002).

[20] A. V. Goltsev, S. N. Dorogovtsev and J. F. F. Mendes, Phys. Rev. E 67, 026123 (2003).

[21] M. Leone, A. Vázques and R. Zecchina, Eur. Phys. J. B 28, 191 (2002).

[22] R. Cohen, K. Erez, D. ben-Avraham and S. Havlin, Phys. Rev. Lett. 85, 5468 (2000).

[23] R. Cohen, K. Erez, D. ben-Avraham and S. Havlin, Phys. Rev. E 66, 036113 (2002).

[24] J. D. Noh, and H. Rieger, Phys. Rev. Lett. 92, 118701 (2004).

[25] J. D. Noh and S.-W. Kim, J. Korean Phys. Soc. 48, S202 (2006).

[26] L. A. Adamic, R. M. Lukose, A. R. Puniyani and B. A. Huberman, Phys. Rev. E 64, 046135 (2001).

[27] S. Lee, S.-H. Yook and Y. Kim, submitted to Nature.

[28] R. Pestor-Satorras and A. Vespignani, Evolution and Structure of the Internet : A Statistical Physics Approach (Cambridge University Press, 2004).

[29] S. Kwon, S. Lee and Y. Kim, condmat/0512372.
[30] L. A. Adamic and B. A. Huberman, science 287, 2115 (2000).

[31] K.-I. Goh, B. Kahng and D. Kim, Phys. Rev. Lett. 87, 278701 (2001). 
(a)

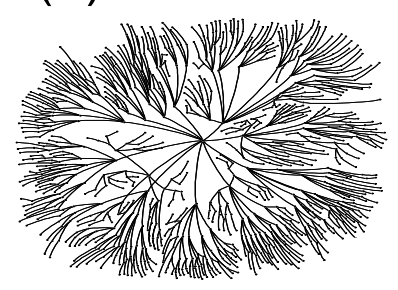

(b)
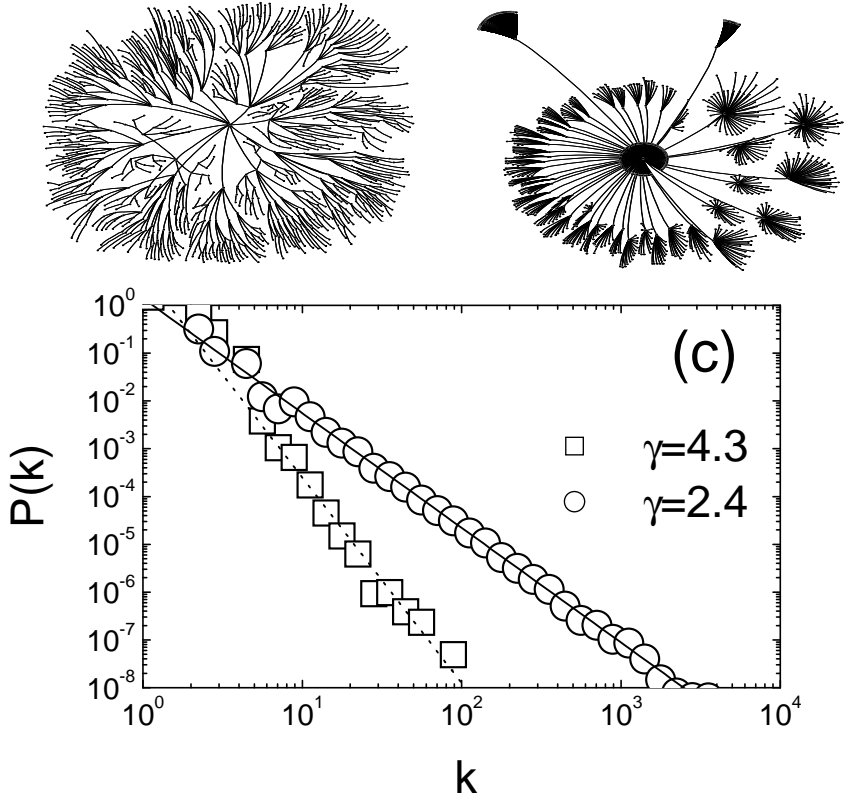

FIG. 1: Schematic structures of TSFNs with $\gamma=4.3$ (a), and 2.4 (b) constructed by the method introduced in Sec. II. The plot of the degree distribution of the TSFNs with $\gamma=2.4$, and 3.0 (c). Network size is $N=10^{5}$. The degree distribution $P(k) \sim k^{-\gamma}$ of the constructed networks follows the original distribution $f(k) \sim k^{-\gamma}$ well. 


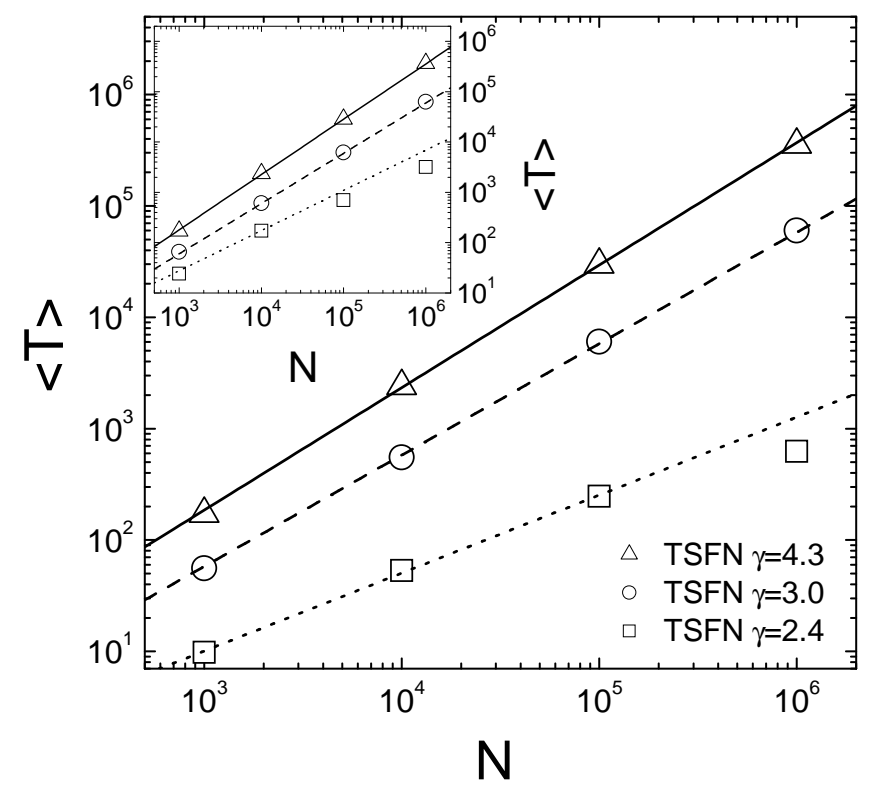

FIG. 2: Average life time $\langle T\rangle$ on TSFNs with $\gamma=4.3,3.0$ and 2.4. The lines represent the best fits of $\langle T\rangle \sim N^{\alpha}$ to data. The obtained exponents are $\alpha \simeq 1.1$ (solid line) for $\gamma=4.3$, $\alpha=1.0$ (dashed line) for $\gamma=3.0$ and $\alpha=0.7$ (dotted line) for $\gamma=2.4$. For $\gamma=2.4\langle T\rangle$ substantially deviates from $\langle T\rangle \sim N^{0.7}$ line when $N=10^{6}$.
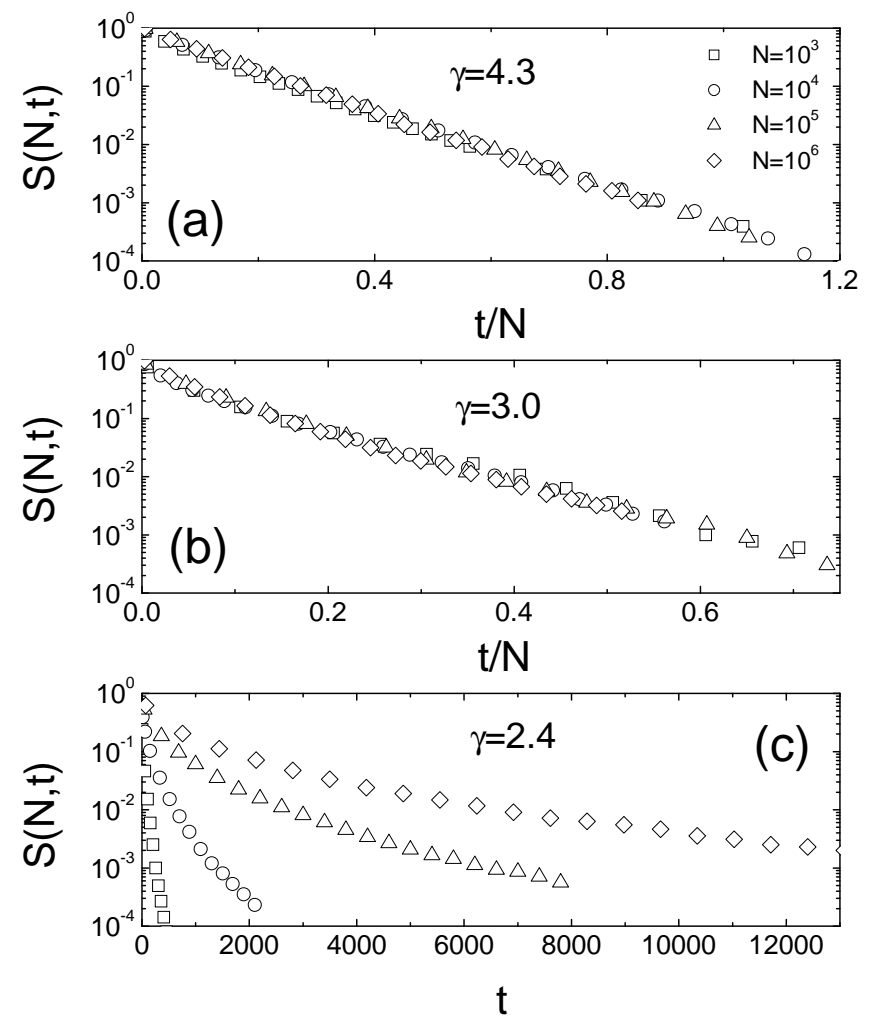

FIG. 3: The scaled plots of the survival probability $S(N, t)$ on TSFNs with $\gamma=4.3$ (a), 3.0 (b) and the plot of $S(N, t)$ for $\gamma=2.4$ (c). For the scaling plots, $\alpha=1$ which is obtained from the fits for $\gamma>3$ in Fig. 2 is used. 


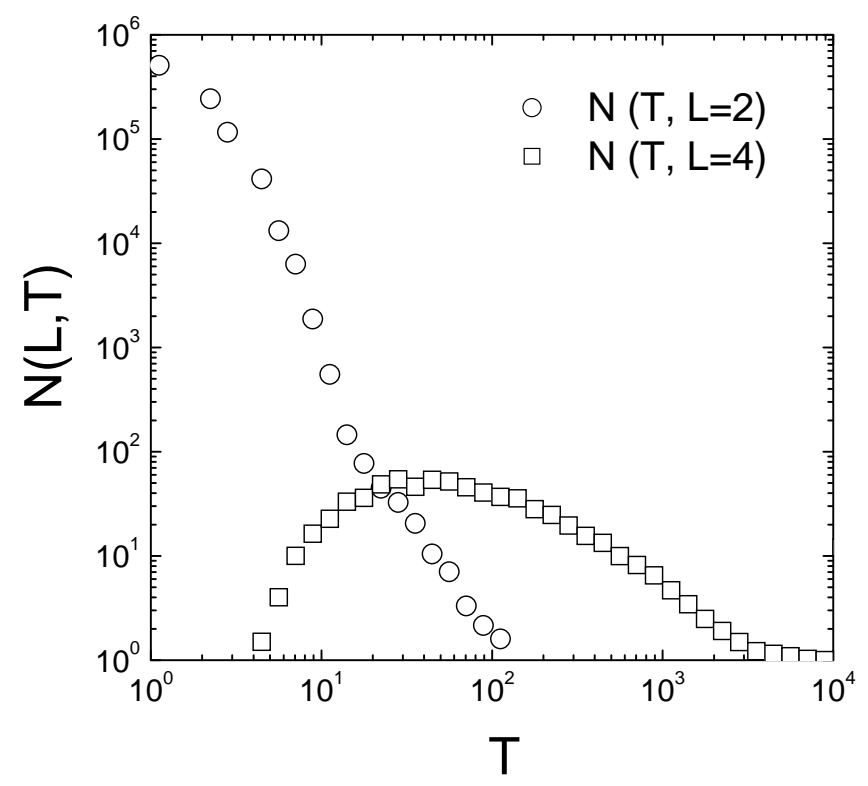

FIG. 4: Plot of $N(L, T)$ on TSFN with $\gamma=2.4$ and $N=10^{4}$. The initial separation $l$ of the lamb and the lion is 2 . $L$ is the maximum separation between the lamb and the lion during random walks (symbols).
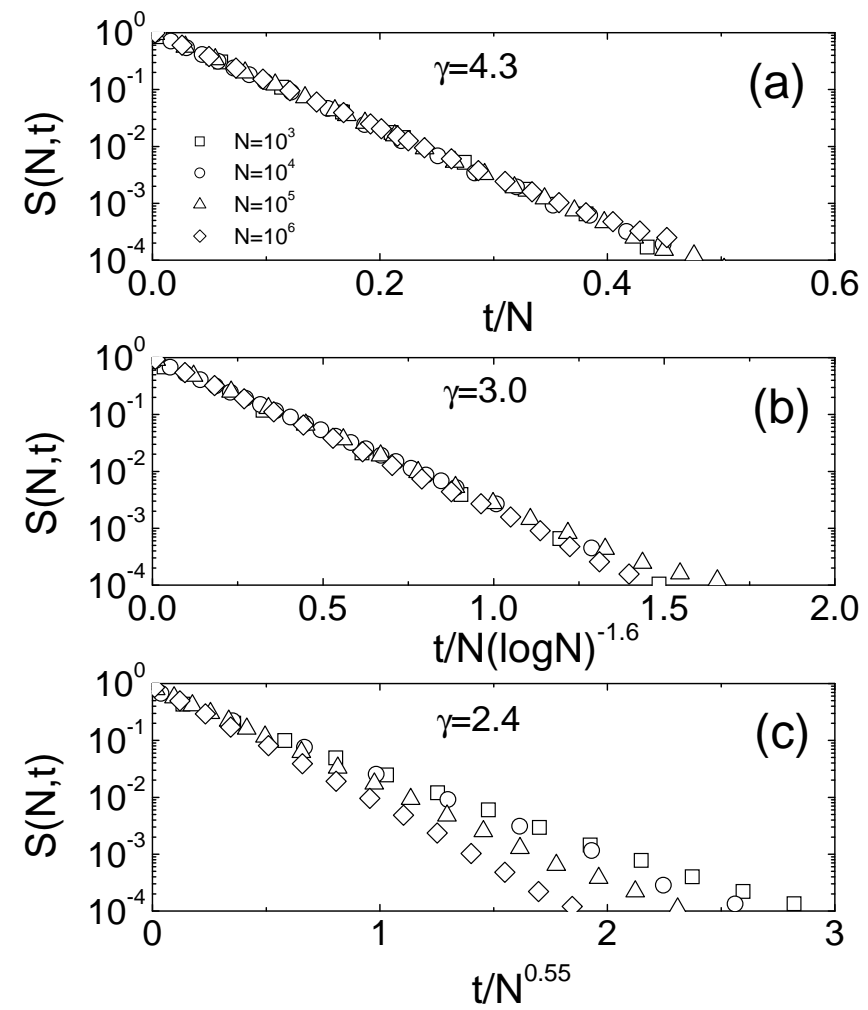

FIG. 5: The scaled plots of the survival probability $S(N, t)$ on LSFNs with $\gamma=4.3$ (a), 3.0 (b), and 2.4 (c). To scale the time $t, \alpha$ 's are obtained from the fits of $\langle T\rangle \sim N^{\alpha}$ to the data (which are not shown in this article). 

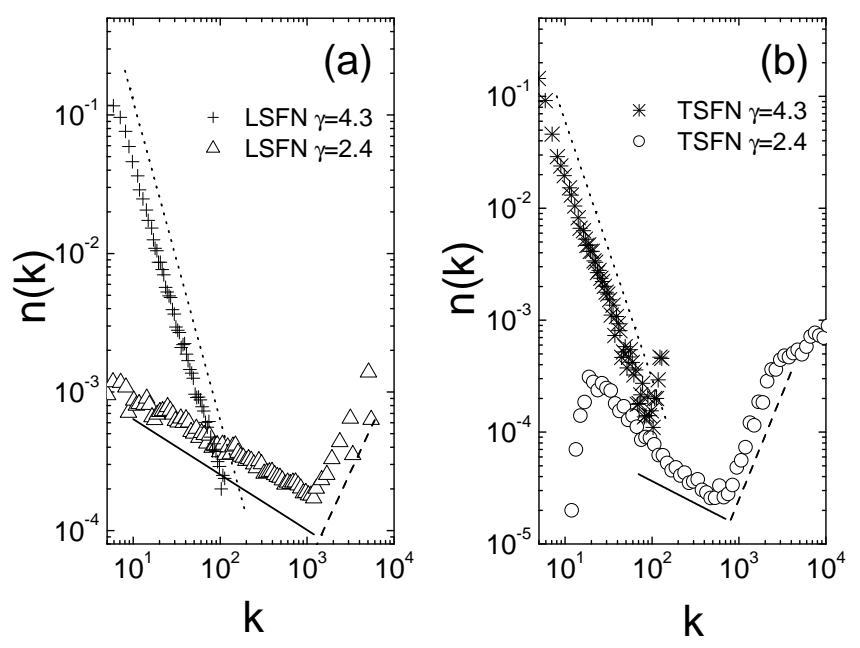

FIG. 6: Plot of capture event distribution $n(k)$ on LSFN (a) and TSFN (b). The lines stand for the relation $n(k) \sim$ $k^{-\sigma}$. The solid lines correspond to $\sigma=0.4$ and the dotted lines represent $\sigma=2.3$. The data for $k<10^{3}$ (a) and for $10^{2}<k<10^{3}$ (b) show good agreements with the relation $\sigma=\gamma-2$. The dashed lines correspond to $\sigma=-1.35$ (a) and $\sigma=-1.9$ (b) representing the anomalous increases of $n(k)$ 's for large $k$. 